\title{
Modal Test Of Six-Meter Hypersonic Inflatable Aerodynamic Decelerator
}

\author{
Nijo Abraham ${ }^{1}$, Ralph Buehrle ${ }^{2}$, Justin Templeton ${ }^{3}$, Mike Lindell ${ }^{4}$ and Sean Hancock ${ }^{5}$ \\ NASA Langley Research Center, Hampton, Virginia, 23681, USA
}

\begin{abstract}
A modal test was performed on the six-meter Hypersonic Inflatable Aerodynamic Decelerator (HIAD) test article to gain a firm understanding of the dynamic characteristics of the unloaded structure within the low frequency range. The tests involved various configurations of the HIAD to understand the influence of the tri-torus, the varying pressure within the toroids and the influence of straps. The primary test was conducted utilizing an eletrodynamic shaker and the results were verified using a step relaxation technique. The analysis results show an increase in the structure's stiffness with respect to increasing pressure. The results also show the rise of coupled modes with the tri-torus configurations. During the testing activity, the attached straps exhibited a behavior that is similar to that described as fuzzy structures in the literature. Therefore extensive tests were also performed by utilizing foam to mitigate these effects as well as understand the modal parameters of these fuzzy sub structures. Results are being utilized to update the finite element model of the six-meter HIAD and to gain a better understanding of the modeling of complex inflatable structures.
\end{abstract}

Keywords: Inflatable, Heat shield, Fuzzy structures, Toroids, Tri-Torus

\section{I.0 INTRODUCTION}

As part of NASA's concept of using inflatable technology for future planetary exploration, the Hypersonic Inflatable Aerodynamic Decelerator (HIAD) is a deployable entry, descent, landing (EDL) system currently in its development phase, consisting of a flexible thermal protection system (FTPS) over a stacked torus inflatable structure (IS) [1]. The tested sixmeter diameter HIAD test article is used to measure the structural responses for comparison with finite element predictions. Over the course of design, the HIAD configuration has evolved from a simple structure of stacked toroids (fabricated from bias braided Technora ${ }^{\circledR}$ and a urethane liner and coating) paired using Kevlar webbing towards a complex structure with added Kevlar radial straps in a crow's-feet arrangement connected to multiple toroids [2] . Additionally, the six-meter test article incorporated removable, rigid buckles into the radial crow's-feet straps to facilitate easy testing of multiple strap configurations. A modified arrangement also includes an additional toroid, named tri-torus (described in Section 2.0). These additions were incorporated to prevent buckling, increase stiffness, and improve the distribution of aerodynamic load, all with a better performance to mass ratio. To understand the dynamic behavior of this complex structure during EDL phase, modal tests were performed on various configurations of this six-meter HIAD to understand the influence of the tri-torus, influence of straps and influence of varying pressure within the toroids.

This paper summarizes the results of the modal tests performed on the six-meter HIAD test article mounted to a fixed base in the high bay area of Building 1250 at the NASA Langley Research Center (LaRC). The frequency range of interest was 5-30 $\mathrm{Hz}$ and the targeted modes were the first four modes.

\subsection{TEST ARTICLE SETUP}

The test article and support structure setup of the HIAD modal test can be seen in Figure 1.

\footnotetext{
${ }^{1}$ Test Engineer, Systems Integration and Test Branch

${ }^{2}$ Branch Head, Systems Integration and Test Branch

${ }^{3}$ Test Engineer, Systems Integration and Test Branch

${ }^{4}$ Structural Analyst, Structural and Thermal Systems Branch

${ }^{5}$ Model Engineer (SSAI), Mechanical Systems Branch
} 


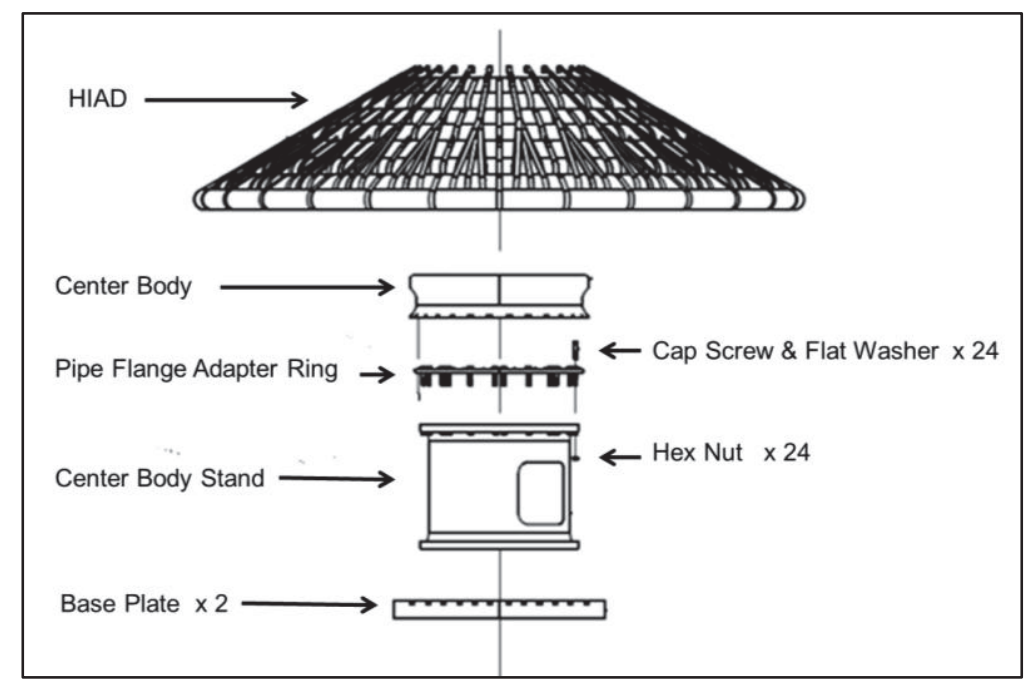

Figure 1 Test Article Setup

The HIAD structure which consists of 8 toroids will be referred in descending order as Toroid 1 or T1 for the top most toroid to Toroid 8 or $\mathrm{T} 8$ for the bottom most toroid, from a nose up position. For the tri-torus configuration, the tri-torus will be referred as Toroid 6.5 or T6.5. The cross-sectional views of configurations with and without the tri-torus are shown in Figure 2 and Figure 3 respectively. Configurations were also tested with the straps and without the straps, as shown in Figure 4 and Figure 5 respectively. The configurations in the order they were tested are given in Table 1.

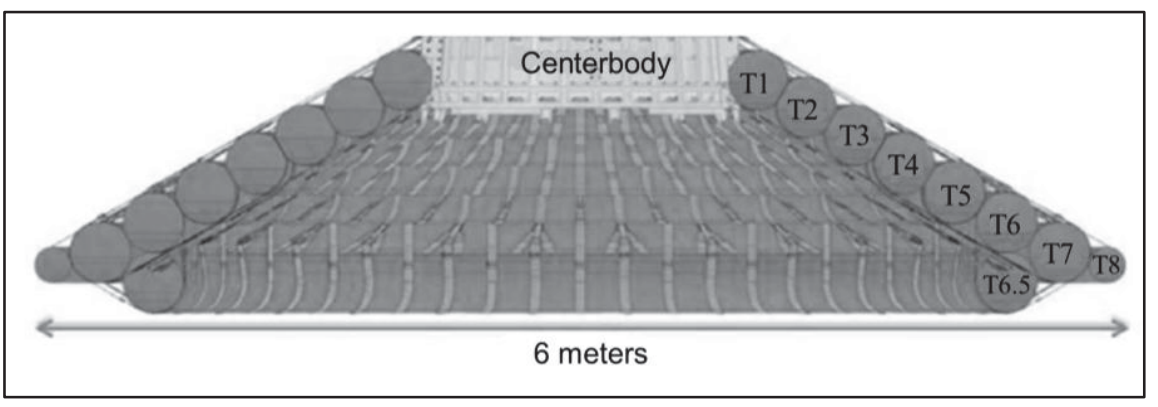

Figure 2 Cross Sectional View - With Tri-Torus Configuration

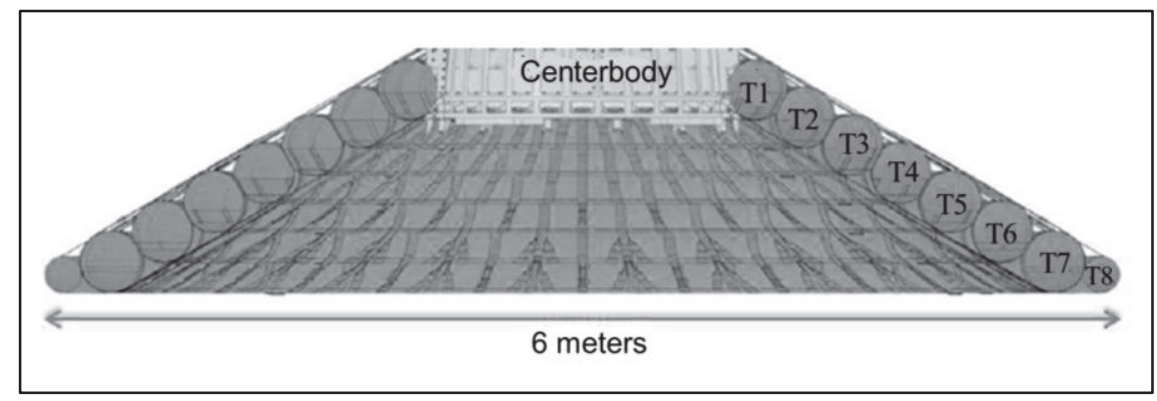

Figure 3 Cross Sectional View - Without Tri-Torus Configuration 


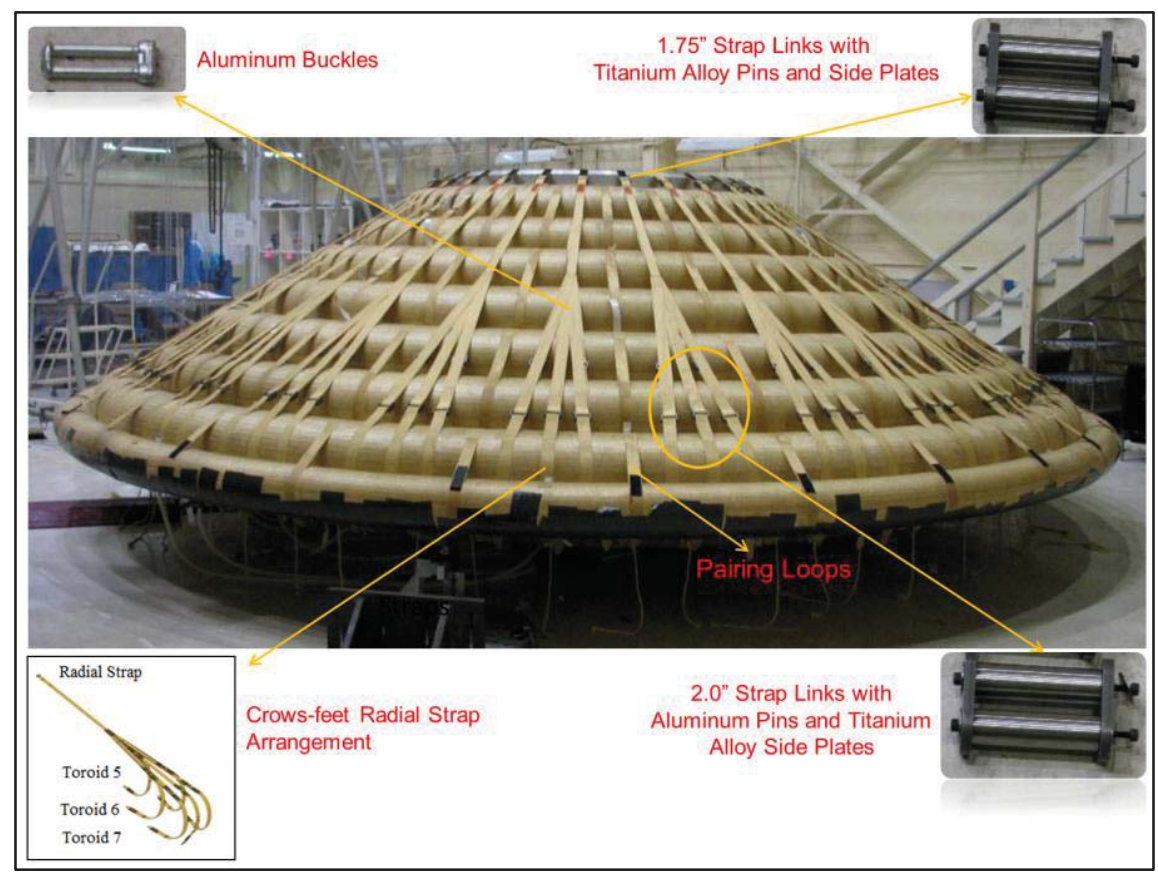

Figure 4 HIAD Configuration with Straps

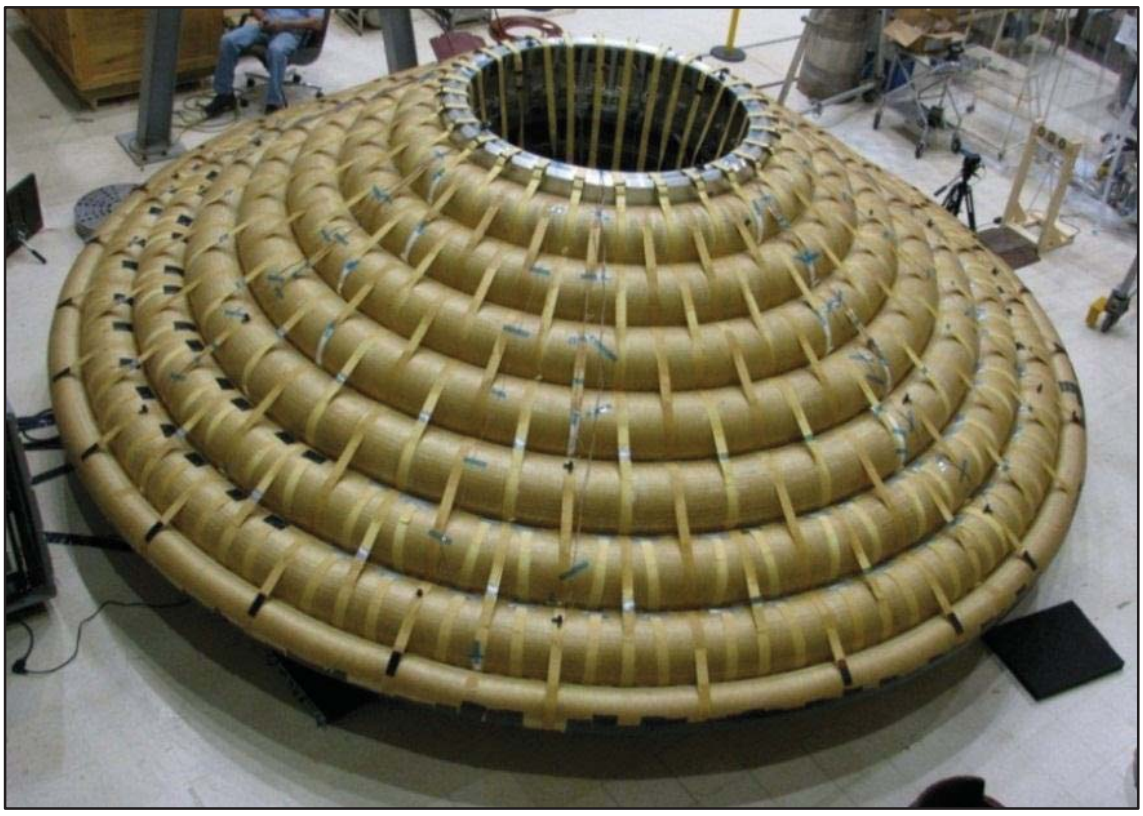

Figure 5 HIAD Configuration without Straps 
Table 1 Test Configuration Matrix

\begin{tabular}{|l|c|l|}
\hline Test Article & Configuration ID & Inflation Pressure \\
\hline \multirow{3}{*}{$\begin{array}{l}\text { Six-meter with Tri-Torus } \\
\text { and Straps }\end{array}$} & 1 & $8 \mathrm{psi}$ all toroids \\
\cline { 2 - 3 } & 2 & $15 \mathrm{psi}$ all toroids \\
\cline { 2 - 3 } & 3 & $20 \mathrm{psi}$ all toroids \\
\cline { 2 - 3 } & 4 & $15 \mathrm{psi}$ T1/T2/T6.5, 8 psi all others \\
\hline \multirow{3}{*}{$\begin{array}{l}\text { Six-meter without Tri-Torus } \\
\text { and with Straps }\end{array}$} & 5 & $8 \mathrm{psi}$ all toroids \\
\cline { 2 - 3 } & 6 & $15 \mathrm{psi}$ all toroids \\
\cline { 2 - 3 } & 7 & $20 \mathrm{psi}$ all toroids \\
\hline \multirow{3}{*}{$\begin{array}{l}\text { Six-meter without Straps } \\
\text { and Tri-Torus }\end{array}$} & 8 & $15 \mathrm{psi}$ T1/T2/T7, 8 psi all others \\
\cline { 2 - 3 } & 9 & $8 \mathrm{psi}$ all toroids \\
\cline { 2 - 3 } & 10 & $15 \mathrm{psi}$ all toroids \\
\cline { 2 - 3 } & 11 & $20 \mathrm{psi}$ all toroids \\
\cline { 2 - 3 } & 12 & $15 \mathrm{psi}$ T1/T2/T7, $8 \mathrm{psi}$ all others \\
\hline
\end{tabular}

\subsection{TEST INSTRUMENTATION}

The instrumentation consisted of 54 accelerometers, PCB series T333B accelerometers, glued with a urethane adhesive to 18 locations of the HIAD test article (shown in Figure 6). Fourteen tri-axial accelerometer assemblies (Locations 1 through 14) were placed on the inflatable and four tri-axial accelerometer assemblies (Locations 15 through 18) on the center body. For each tri-axial accelerometer assembly, the first accelerometer pointed radially outward, the second accelerometer pointed tangential to the toroid and the third accelerometer pointed in the normal direction of Figure 6 . The transducers were placed on pre-determined locations to capture the desired first four modes.

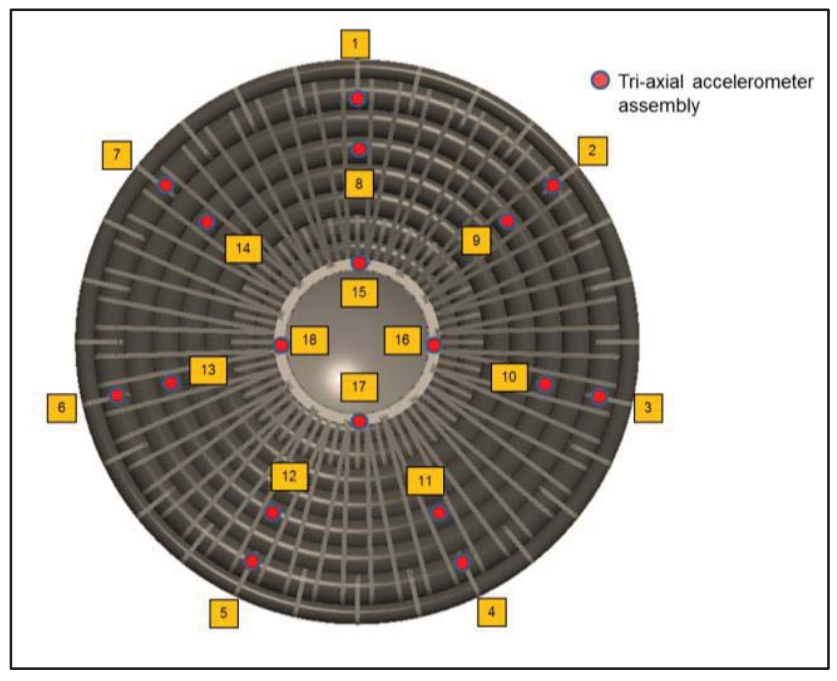

Figure 6 Top View of the Accelerometer Assembly Locations

\subsection{EXCITATION TECHNIQUES}

The excitation locations were under Toroid 7 beneath the accelerometer assemblies 2 and 6 . The primary excitation of the structure was conducted using eletrodynamic shakers. The mounting block for each shaker was oriented at an angle of 30 degrees from vertical to excite both the vertical and torsion modes (shown in Figure 7). 


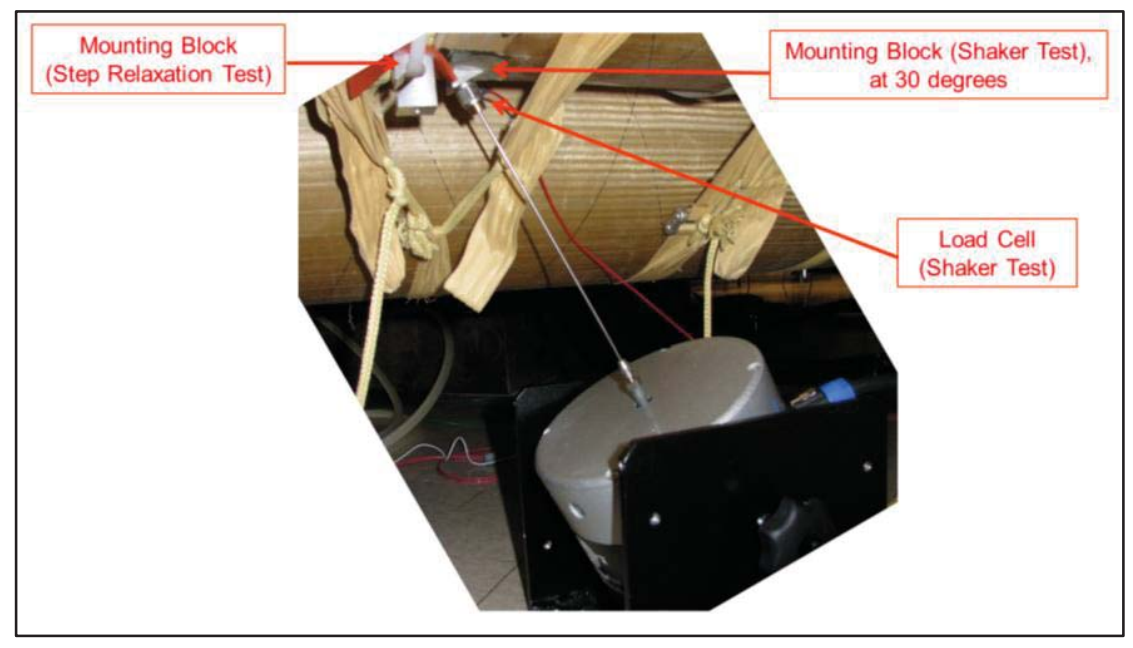

Figure 7 Shaker Setup

To verify that the modal parameters of this complex structure were not affected by the stiffness of the stinger rods, the shaker test results were compared with the results obtained using a step relaxation technique. The test setup for the step relaxation technique is shown in Figure 8, where the load is released and the decaying responses were recorded. The modal parameters were obtained from the step relaxation techniques using the System/Observer/Controller Identification Toolbox (SOCIT) which utilizes a system identification process to calculate the mathematical representation of a physical system using experimental free decay time records [3]. The step relaxation technique was only performed for the configurations with the tri-torus (Configurations 1 through 4). The difference in the modal frequencies between the shaker and step relaxation test techniques were seen to range from 0.1 - 3\%, without any significant trends. This percent difference was acceptable for this study. It should be noted that all the results given in this paper are from the shaker tests.

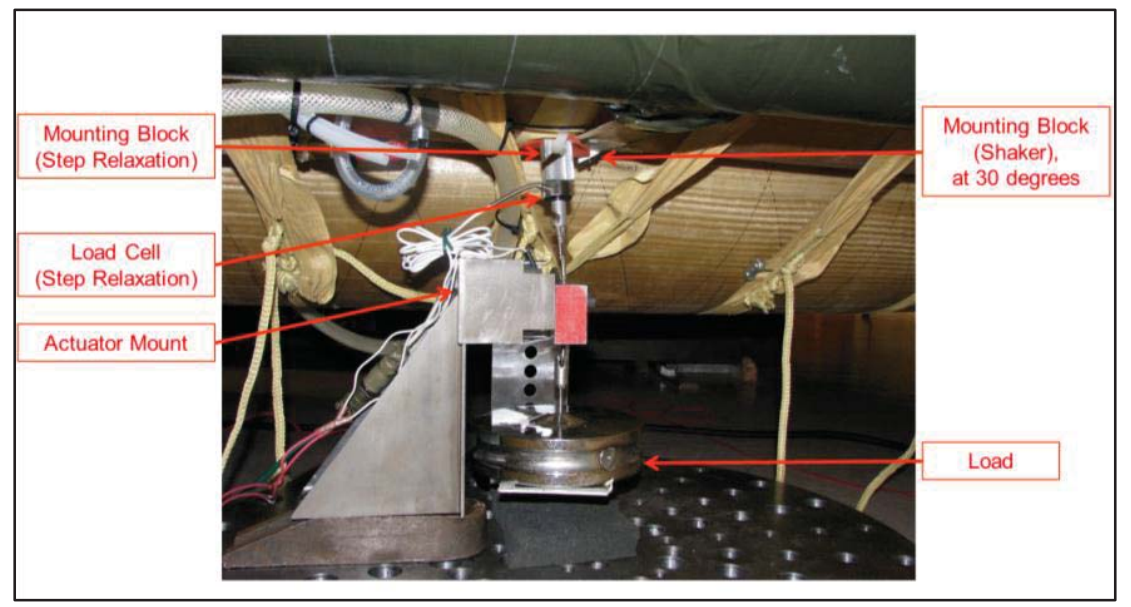

Figure 8 Step Relaxation Setup

\subsection{MODAL TEST RESULTS}

The modes obtained from the experimental results are shown in their respective order in Figure 9. With reference to Figure 1, the brown quadrilateral represents the bottom plane of the center body assembly. The black quadrilateral represents the baseplate. The gray quadrilateral represents the four tri-axial accelerometer assembly locations for the center body. Finally, the joints of the orange quadrilaterals represent the fourteen tri-axial accelerometer assembly locations on the inflatable. 


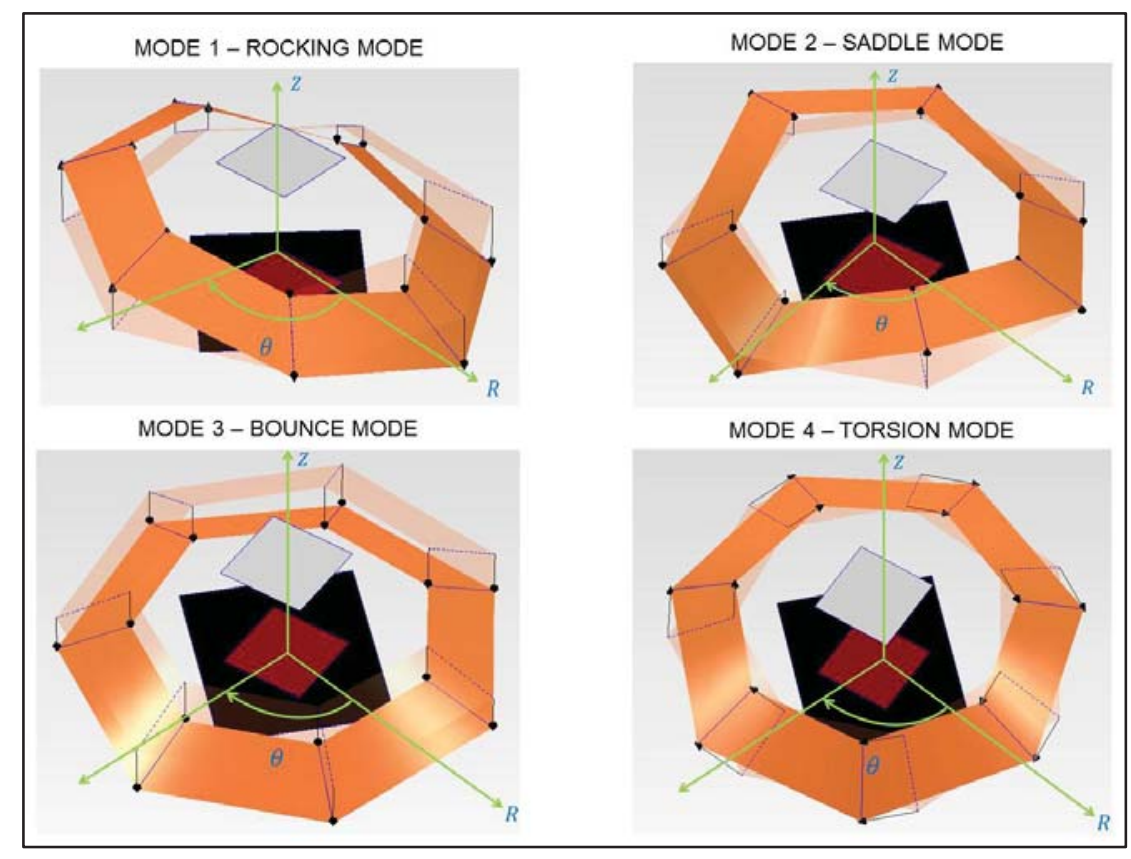

Figure 9 Order of Targeted Modes

\subsection{PREDICTED VS. EXPERIMENTAL RESULTS}

Results from the experimental tests and its comparison with the predicted values are tabulated in Table 2. It was observed that the percentage difference between the frequencies of the first two modes was less than $4 \%$ but between the third and fourth mode was 33-35\%. Data is currently being analyzed to determine the cause for this difference to obtain an accurate finite element model.

Table 2 Percentage Difference between Predicted and Experimental Modal Frequencies

\begin{tabular}{|c|c|c|c|}
\hline \multirow{3}{*}{ 20 psi - With Tri-Torus, With Straps and Foam Configuration } \\
\hline \multirow{2}{*}{ Mode } & Predicted & Experimental & $\begin{array}{c}\text { \% difference } \\
\text { Experimental - Predicted }\end{array} \times 100$ \\
\hline & $\mathrm{Hz}$ & $\mathrm{Hz}$ & $\%$ \\
\hline Rocking & 8.50 & 8.77 & 3.07 \\
\hline Saddle & 12.30 & 12.26 & -0.32 \\
\hline Bounce & 22.10 & 16.37 & -35.00 \\
\hline Torsion & 14.50 & 21.68 & 33.11 \\
\hline
\end{tabular}

\subsection{INFLUENCE OF THE STRAPS}

The function of the radial and crow's feet strap is to distribute the aerodynamic load from the outer toroids directly to the center-body to alleviate the loads carried by Toroid 1 and Toroid 2. Figure 10 shows a comparison of the imaginary portions of the frequency response functions for configurations with and without straps referred to as configuration 7 and 11 (see Table 1) respectively. Since the frequency response functions for the radial and vertical accelerometer locations resulted in modes at the same frequencies, only the responses from the vertical and tangential accelerometers will be plotted for all the figures within this paper. 


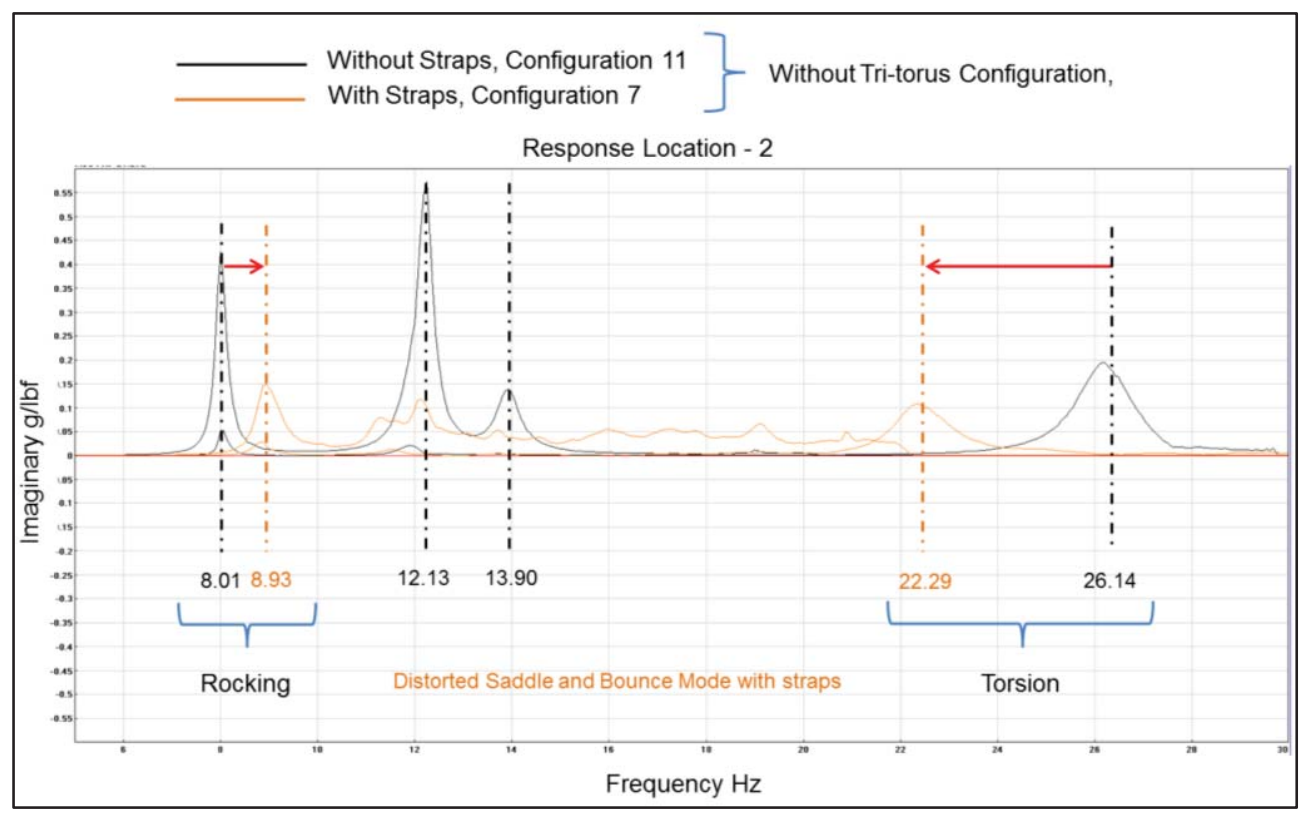

Figure 10 FRFs highlighting Influence of Straps

It is observed that the straps caused an increase in the natural frequency for the rocking mode whereas it decreased the natural frequency for the torsion mode. The increase in the frequency of the rocking mode is due to the increase in the stiffness of the structure caused by the straps. For the torsion mode, the larger mass concentration of the strap links (shown in Figure 4) located at a larger distance from the structure's center of mass is the likely cause for the decrease in the torsion mode's natural frequency resulted by the increase in the rotational moment of inertia.
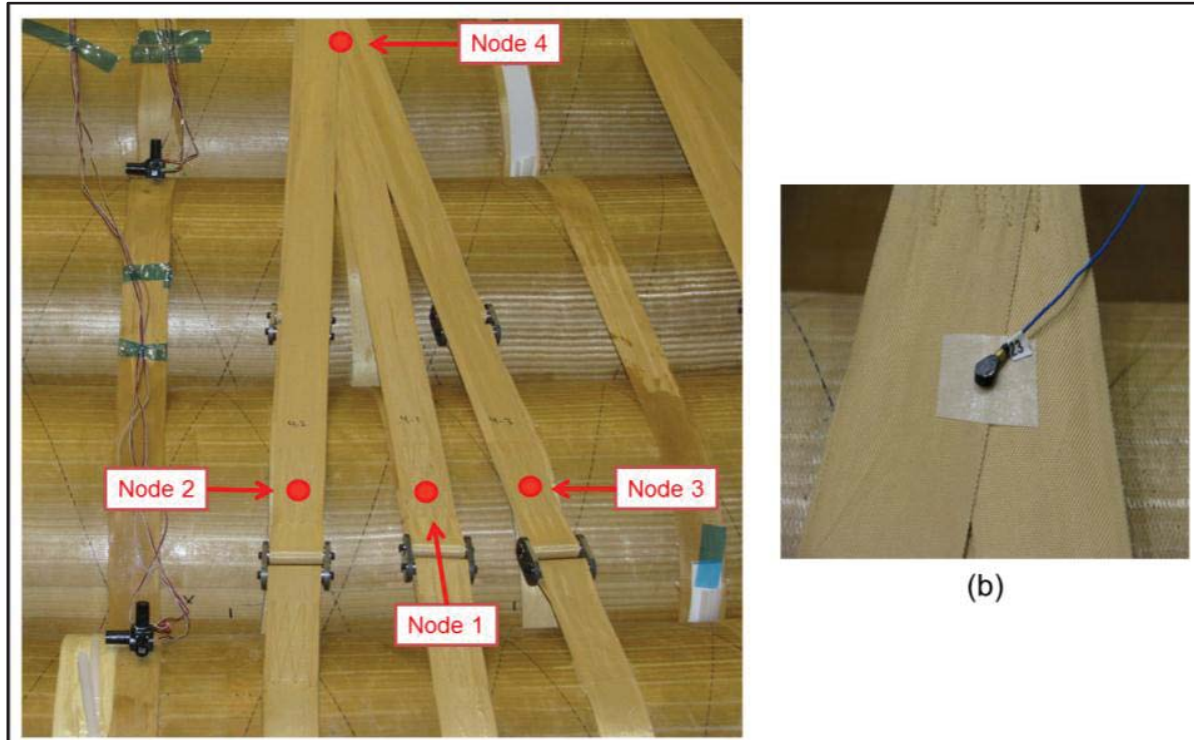

(b)

(a)

Figure 11 Tear-drop Accelerometer Installations; (a) Locations on the Strap and (b) Tear-drop Accelerometer on the Strap

One other observation is that the straps caused a distortion within the frequency range where the saddle and bounce modes were expected. Therefore, to find the dynamic characteristics of the straps, four PCB 352C22 teardrop accelerometers were placed on four locations within a radial (shown in Figure 11). Nodes 1, 2 and 3 were the locations where most relative displacement of the straps was noticed during vibration. Figure 12 shows the imaginary part of the frequency response 
functions of the individual straps superimposed with that of the structure's response for configuration 7 (see Table 1). These modal results showed that the distortion of the structure's modes was caused by the local modes of the straps. Node 4 did not have any local modes within the frequency range of interest.

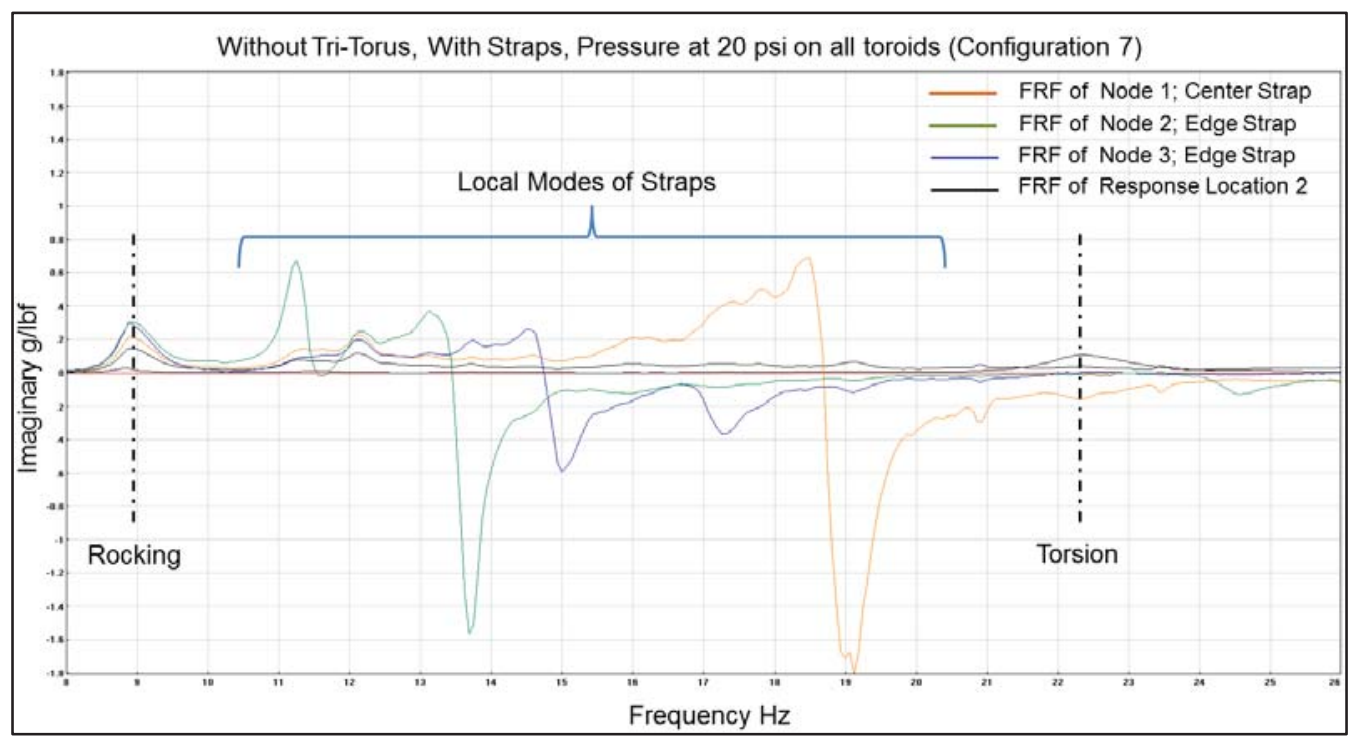

Figure 12 FRFs highlighting Local Modes of Straps

The distortion phenomenon caused by the straps can be explained by considering the inflatable structure with the straps as a fuzzy structure with the straps acting as fuzzy sub structures [4]. The inflatable by itself, can be considered as the master structure. The local modes are being excited as a result of energy transfer to fuzzy substructures through the common boundary between the master structure and fuzzy structure. This leads to dissipation at the global mode frequencies of the inflatable structure (master structure). Therefore, due to this fuzzy structure phenomenon, modeling the straps as discrete spring mass models will not capture the resulting local modes caused by the straps. One has to take into account the internal degrees of freedom of the straps during finite element modeling. One possible solution of capturing these effects of straps is by using probabilistic fuzzy boundary impedance elements [5] at the common boundary between the inflatable and straps.

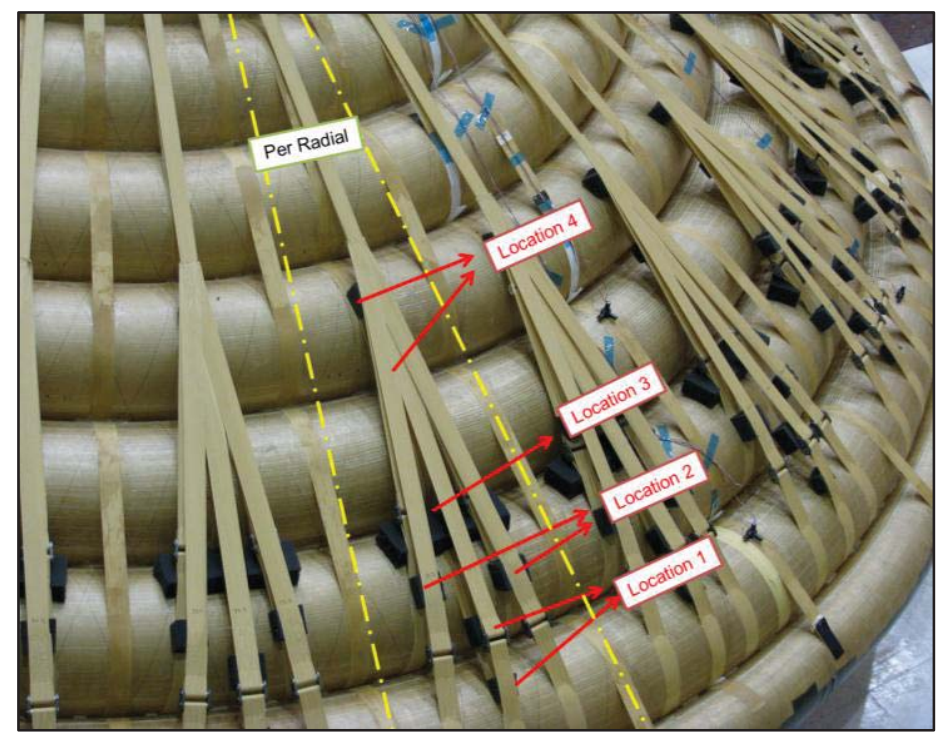

Figure 13 Foam Locations per Strap Radial 
Since modeling of straps as fuzzy structures can be challenging, another solution would be to increase the stiffness of the straps such that the frequencies of the distorting local modes would increase beyond the frequency range of interest. It was also desired to increase damping at these strap locations to decrease their vibration amplitude. These were achieved by placing foam blocks at selective locations such that the straps were isolated from the structure. Figure 13 shows the locations where the foam blocks were placed; Location 1 targets the central strap and its buckles attached to Toroid 7, Location 2 targets the targets the end straps and its buckles attached to Toroid 7, Location 3 targets the mid-strap assembly and its buckles attached to Toroid 6 and Location 4 isolates the strap assemblies attached to Toroid 7 from that of Toroid 6 .

After placing the foam, no distortion of modes was observed within the targeted frequency range. The comparison of the imaginary part of the response functions between the strap configurations with and without foam (shown in Figure 14) show an increase in the structure's stiffness at the bounce mode possibly caused due to tightening of the straps.

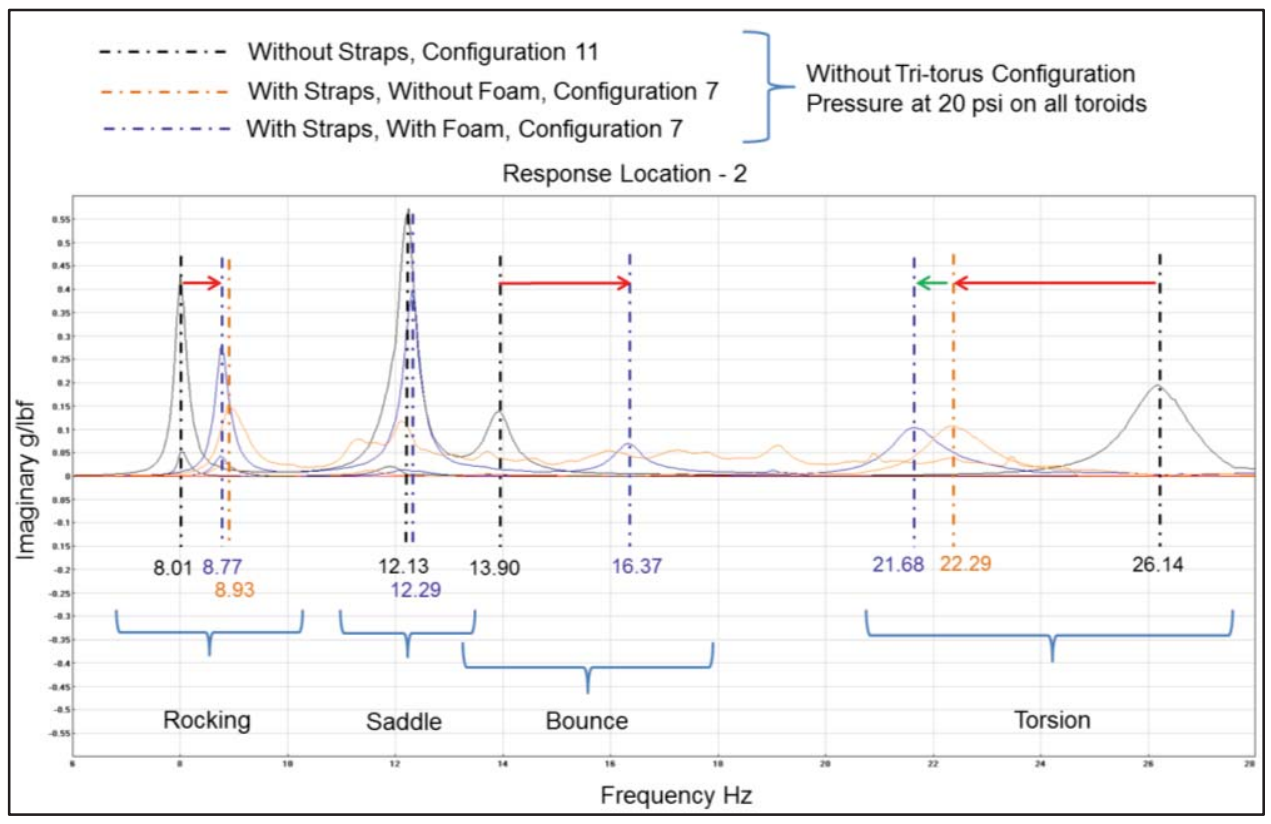

Figure 14 FRFs highlighting Influence of Foam

\subsection{INFLUENCE OF TRI-TORUS}

The function of the tri-torus is to increase the stiffness of the structure as well as prevent buckling of the toroids observed during static tests. Figure 15 shows a comparison of the imaginary part of the frequency response functions for configurations 3 and 7 (see Table 1), with and without the tri-torus respectively. One of the observations is that the tri-torus decreased the natural frequencies of the rocking, bounce and torsion modes when compared to the configuration without tri-torus. This is possibly due to the mass of the tri-torus, which is at a larger distance from the structure's center of mass resulting in a predominant mass effect for these three modes, causing a decrease in the natural frequency. On the other hand, the tri-torus was observed to cause an increase in stiffness affecting only the natural frequency of the saddle mode where the tri-torus would undergo bending deformation.

Another observation that can be made from Figure 15 is that the tri-torus configuration resulted in the rise of coupled modes due to the combined stiffness and mass effect it had on the saddle and bounce modes respectively. At a higher pressure of 20 psi, the individual modes within the coupled mode could be distinctively identified contrary to the lower pressures where the modes were harder to distinguish. This is shown in Figure 16 based on the tests conducted on configurations 4 and 8 (see Table 1). 


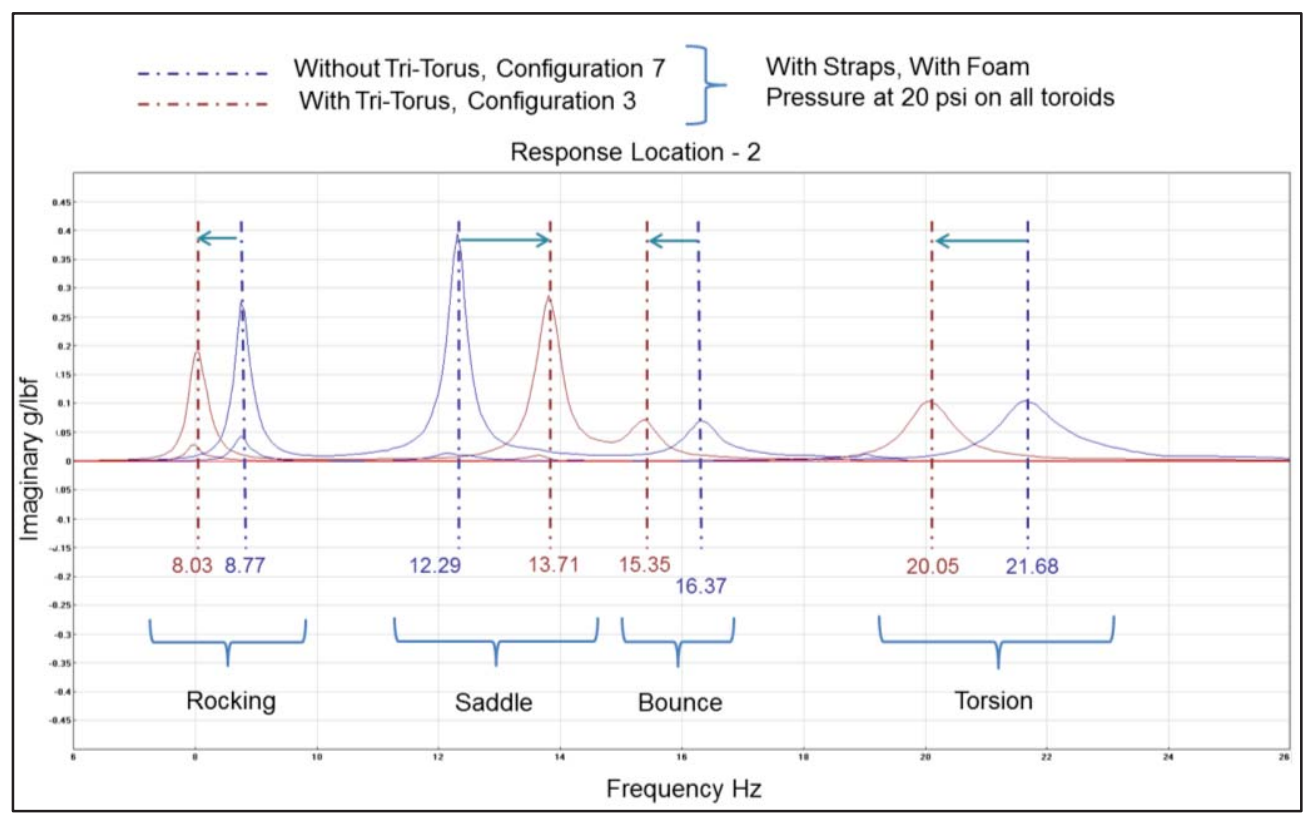

Figure 15 FRFs highlighting Influence of Tri-torus at 20 psi Pressure Configuration

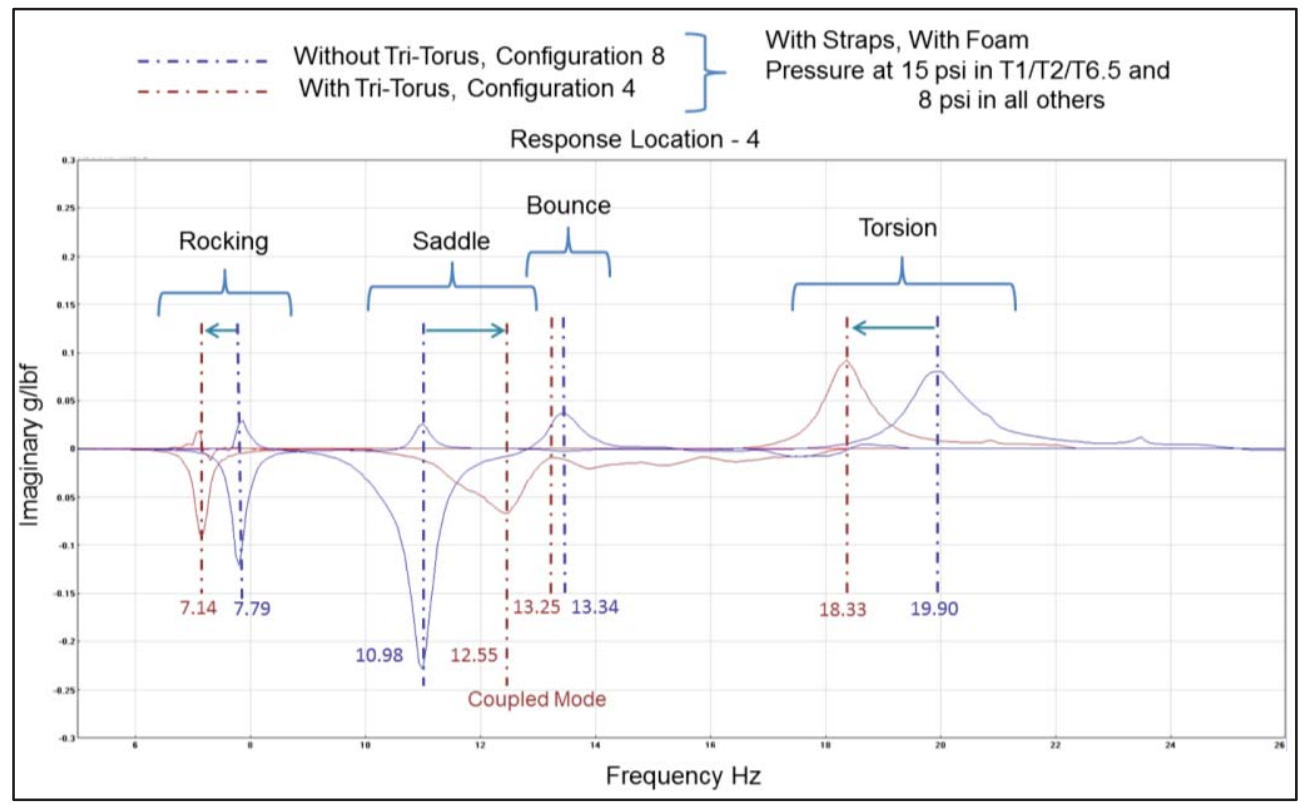

Figure 16 FRFs highlighting Coupled Modes at 15/8 Pressure Configuration

\subsection{COMPARISON OF TRI-TORUS AND STRAPS INFLUENCES}

Figure 17 shows the influences of the straps and tri-torus on frequency response functions at the 20 psi pressure configuration. Similar behavior was seen for all the other pressure configurations as well. It was observed that

1) The straps caused predominantly a stiffness effect, whereas the tri-torus resulted in a mass effect. In other words:

a. straps caused an increase in natural frequency for three of the four target modes whereas

b. tri-torus caused a decrease in natural frequency for three of the four target modes

2) The rocking mode was influenced by the stiffness effect of straps and almost equal mass effect of tri-torus, thereby causing no significant resultant effect 
3) The saddle mode was primarily influenced by the stiffness effect of the tri-torus.

4) The bounce mode was influenced by the stiffness effect of the straps and the mass effect of the tri-torus, with the straps having a predominant influence

5) The torsion mode was influenced by the mass effects of both straps and tri-torus, with the straps having a predominant influence.

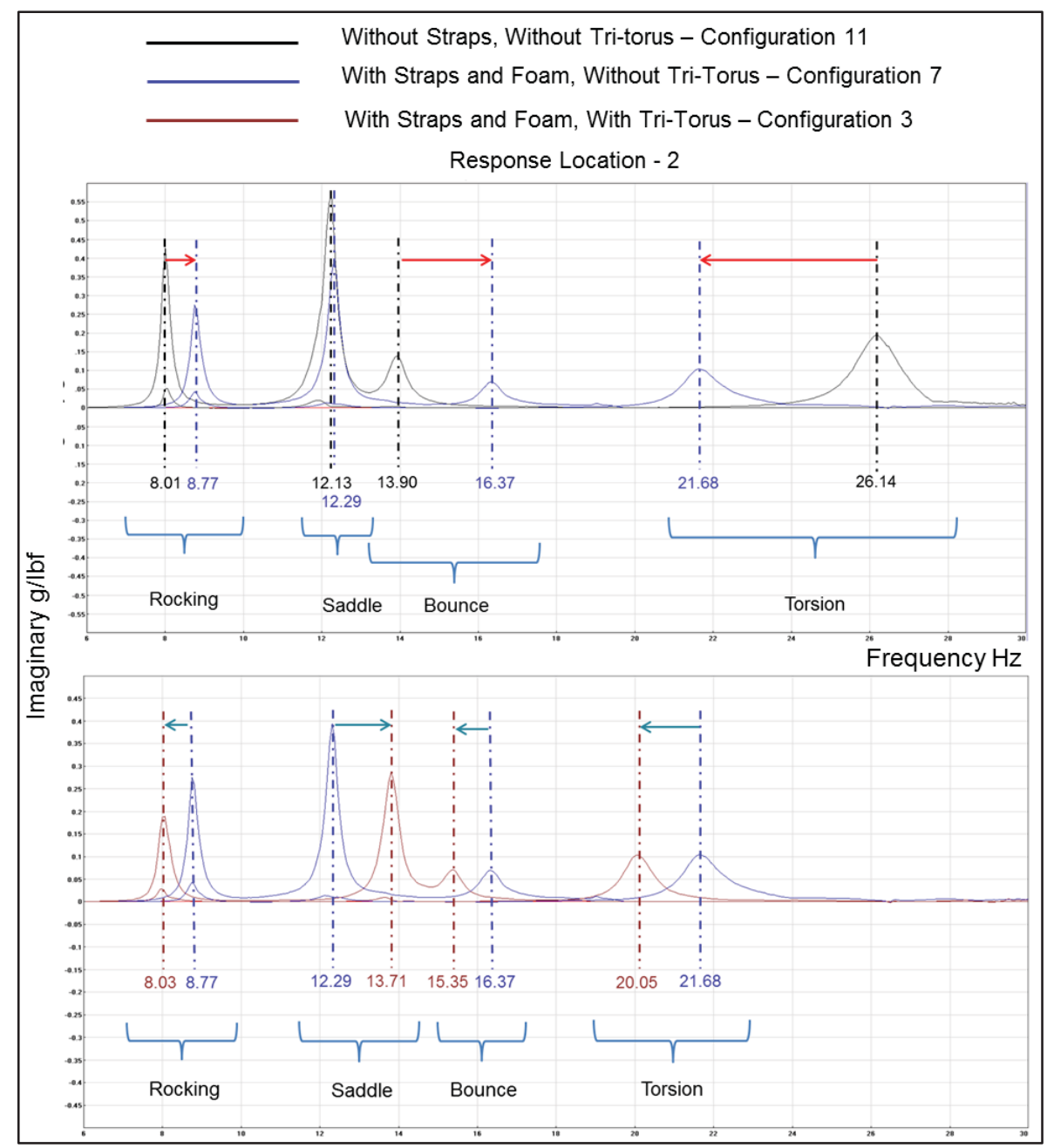

Figure 17 Comparison of FRFs highlighting Influence of Straps and Influence of Tri-torus

\subsection{INFLUENCE OF VARYING TOROID PRESSURE}

The effects of toroid pressure were examined with the configuration series 1-4, 5-8 and 9-12 (see Table 1). The behavior of the structure was similar across all these series. Figure 18 shows the imaginary portion of the frequency response functions for configuration series 1-4 with foam. It was observed that the natural frequencies of the structure increase with increasing pressure within the toroids. This is due to the fact that the pressure increases the stiffness of the structure, and since stiffness is directly proportional to the natural frequency squared, the natural frequency of the structure increases as well. This phenomenon is seen throughout all the modes. The natural frequency did not show any proportional increase with respect to the increase in pressure; however the percentage change in frequency between pressures remained approximately the same for all modes other than the bounce mode. Natural frequency squared is also inversely proportional to the mass. However, in this case, the increase in mass due to the increase in pressure is negligible compared to the increase in stiffness. 


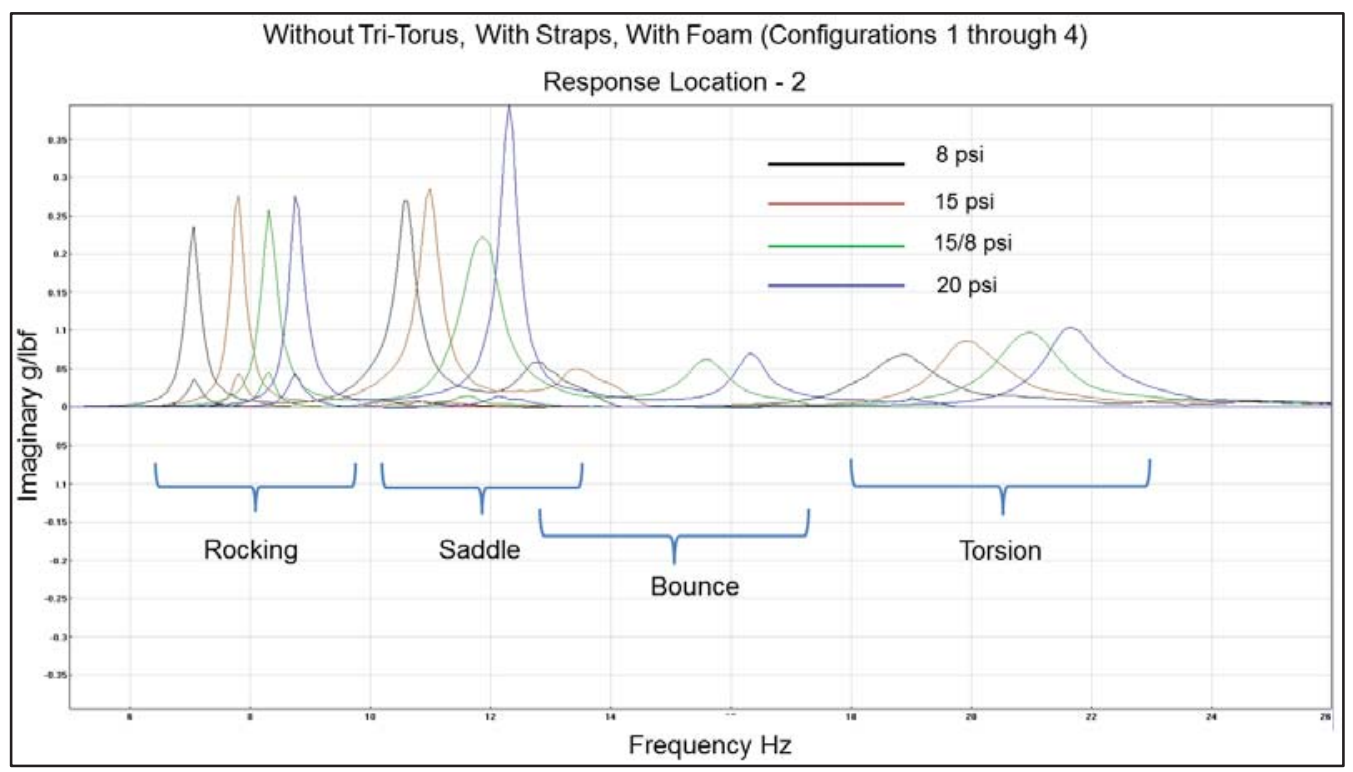

Figure 18 FRFs highlighting Influence of Pressure

\subsection{CONCLUSIONS}

The modal test successfully identified the first four modes for the HIAD structure. The tests were able to identify the influence of the straps and tri-torus on the structure's modal parameters. It showed that the tri-torus predominantly results in a mass effect whereas the straps predominantly result in a stiffness effect on the modal frequencies of the structure. This is in tandem with the intent of the tri-torus to increase rigidity thereby preventing buckling as seen in the saddle mode. It was observed from the results that the stiffness of the structure increased with increasing toroid pressures. Also observed were the rise of coupled modes with the tri-torus configuration and the behavior of the unloaded straps as fuzzy substructures. To summarize, these tests provided substantial information on the dynamics of complex inflatable structures. These results are being used to verify and update the finite element modeling techniques for these HIAD systems. The data can also be used to aid controller design for the reentry trajectory control system of future HIAD flight articles. Future test plans include the investigation of dynamic effects of: scaling through testing of different size test articles, the addition of thermal protection systems, and simulated aerodynamic load.

\subsection{REFERENCES}

[1] S. J. Hughes, M. F. Cheatwood, A. M. Calamino and H. S. Wright, "Hypersonic Inflatable Aerodynamic Decelerator Technology Development Overview," in AIAA 2011-2524, 21st AIAA Aerodynamic Decelerator Systems Technology Conference and Seminar, Dublin, Ireland, 23-26 May 2011.

[2] G. T. Swanson, A. M. Cassell, K. R. Johnson, S. J. Hughes, A. M. Calamino and M. F. Cheatwood, "Structural Strap Tension Measurements of a 6 Meter Hypersonic Inflatable Aerodynamic Decelerator under Static and Dynamic Loading," in AIAA Aerodynamic Decelerator System (ADS) Conference, Daytona Beach, Florida, 25-28 March 2013.

[3] J.-N. Juang, Applied System Identification, Englewood Cliffs, New Jersey, 07632: Prentice Hall, Inc., 1994.

[4] C. Soize, "Vibration Damping in Low Frequency Range due to Structural Complexity. A Model based on the Theory of Fuzzy Structures and Model Parameters Estimation," Computers and Structures Vol. 58, No. 5, pp. 901-915, 1995.

[5] C. Soize, "Probablistic Structural Modeling in Linear Dynamic Analysis of Complex Mechanical Systems. I-Theoretical Elements," La Recherche Aerospatiale (English Edition), 5, pp. 23-48, 1986. 